doi: 10.52370/TISC2194BK

\title{
ADAPTIVE NEURO FUZZY ESTIMATION OF THE OPTIMAL COVID-19 PREDICTORS FOR GLOBAL TOURISM
}

\author{
Boris Kuzman ${ }^{1}$; Biljana Petković ${ }^{2}$
}

\begin{abstract}
COVID-19 is a pandemic that has emerged as a result of 2019-novel coronavirus droplet infection (2019-nCoV). Recognition of its risk and prognostic factor is critical due to its rapid dissemination and high casefatality rate. Tourism industry as one of the greatest industries has suffered a lot in the pandemic situation. The main aim of the study was to present travelers' reaction during the pandemic by data mining methodology. The effect of eleven predictors for COVID-19 was also analyzed. The used predictors are: population density, urban population percentage, number of hospital beds, female and male lung size, median age, crime index, population number, smoking index and percentage of females. As the output factors, infection rate, death rate and recovery rate were used. The analyzing procedure was performed by adaptive neuro fuzzy inference system (ANFIS). The results revealed that the frequency of the used words in the pandemic show the highest impact on the travelers' reactions. Number of hospital beds and population number is the optimal combination for the best prediction of infection rate of COVID-19.
\end{abstract}

Key Words: COVID-19, Tourism industry, Predictive analytics, Hybrid model, predictors

JEL classification: $R 58, R 59$

\section{Introduction}

Since December 2019, a novel coronavirus (SARS-CoV-2)-infected pneumonia (COVID-19) has been circulating in Wuhan and has quickly

\footnotetext{
${ }^{1}$ Boris Kuzman, Professor, Institute of Agricultural Economics, 15 Volgina Street, 11060 Belgrade, Serbia, 063299111

${ }^{2}$ Biljana Petković, Ph.D student, University of Educons, Business Economics, Vojvode Putnika 85-87, 21208 Sremska Kamenica, Serbia, 0655221763
} 
spread throughout China. Predicting the Coronavirus outbreak, which has spread to over 200 countries and has already been declared a pandemic by the World Health Organization, is a difficult challenge. Situational demand is an effective predictor of the unpredictable gruesomeness to ensure better healthcare service management. Demonstrating and forecasting COVID19 in orders with minimal knowledge structures becomes a difficult task.

In the Wang et al. study, (2020b) it has been suggested that NCD4LR is a potential and useful biomarker for predicting the virus negative conversion time in COVID-19 patients. A hybrid model that incorporates ensemble empirical mode decomposition (EEMD) and artificial neural network (ANN) for predicting the COVID-19 epidemic has been proposed in an article by Hasan (2020) where the result of this investigation showed that the proposed model outperforms compared with traditional statistical analysis. Many laboratory indicators, such as neutrophils, AST, GT, ALP, LDH, NT-proBNP, Hs-cTnT, PT, APTT, D-dimer, IL-2R, IL-6, IL-8, IL$10, \mathrm{TNF}, \mathrm{CRP}$, ferritin and procalcitonin, were all significantly increased in deceased patients compared with recovered patients on admission (Wang et al., 2021a).

In a large cohort of COVID-19 patients of European origin, main risk factors for mortality were older age, comorbidities, low lymphocyte count and high Radiographic Assessment of Lung Edema (RALE) (Fabio et al., 2020). The aim of the research conducted by Xie et al., (2020) was to develop a quantitative method for clinicians to predict the probability of improved prognosis in patients with coronavirus disease 2019 (COVID19). The impact of obesity on the prognosis and disease severity of COVID-19 has been explored in an article by Tamara \& Tahapary (2020) where it was found that obesity is an independent risk and prognostic factor for the disease severity and the requirement of advanced medical care in COVID-19.

Elevated Lactate dehydrogenase (LDH) levels were associated with a 6fold increase with regards to developing severe disease and a 16-fold increase with regards to mortality in patients with COVID-19 (Henry et al., 2020). Totally, 36 clinical indicators significantly associated with severe/critical symptom for COVID-19 were identified (Sun et al., 2020b). Lymphopenia and eosinopenia may serve as predictors of disease severity and disease progression in the COVID-19 patients, and enhancing the cellular immunity may contribute to COVID-19 treatment (Sun et al., 2020a). In a paper by Zhang et al., (2020) a segmented Poisson model was 
employed to analyze the available daily new cases data of the COVID-19 outbreaks and the analysis allowed to make a statistical prediction on the turning point, the duration and the attack rate.

There are currently no reliable methods for predicting COVID-19's outcome. The main aim of the study was to present travelers' reaction during the pandemic by data mining methodology. It has also established a model for predicting the prognosis of the disease based on the selection of the most impactful predictors. One of the most widely used approaches for modeling and simulation of various structures and processes is artificial neural networks. Since artificial neural networks have parallel architectures for solving complex and highly nonlinear problems, this is the case. Therefore, the main aim of the study is to apply artificial neural network merged with fuzzy logic for analyzing of COVID-19 predictors. The used predictors are: population density, urban population percentage, number of hospital beds, female and male lung size, median age, crime index, population number, smoking index and percentage of females. As the output factors infection rate, death rate and recovery rate were used. Adaptive neuro fuzzy inference system (ANFIS) (Jang, 1993; Laković et al., 2021; Milić et., 2021; Petković et al., 2021a, 2021b, 2021c;) is used as a type of artificial neural network which is suitable for nonlinear data samples.

\section{Methodology}

\section{COVID-19 predictors}

In order to enable precise and reliable application of the ANFIS methodology there is a need to establish a database with good quality and acceptable size. The data size depends on the data quality. In other words, if the data quality is good then there is no need to increase size of the database. Therefore, the literature review in this study was chosen in order to establish a reliable database.

The TF-IDF is a statistical measure that assesses the relevance of a word to a document in a set of documents. This is accomplished by multiplying two metrics: the number of times a word appears in a document and the word's reciprocal document frequency across a set of documents. The main point is to create a large image of frequently used words. Since the word frequency analysis requires data on individual tokens, even though words have a high frequency, they cannot produce a meaningful big picture. The 
most commonly repeated words in this study are simple words that also reveal the dataset's thematic structure. When you look at the figure, you'll notice that, PEOPLE, TRAVEL, VIRUS, DAY, CASE, TIME, CANCEL, and TRIP stand out. Any of these terms occur more than 3000 times a year. As a result, even this small number of tokens could mean that the majority of users tend to cancel their trips. The expressions FLIGHT, WEEK, CORONAVIRUS, CHINA, POST, COUNTRY, and GOOD were repeated in the frequency range of 2000-3000.

Table 1 shows input parameters used in the study for covid-19 predictors while Table 2 shows output parameters which are paired with the input data. The used input predictors are: population density (World Population Review, 2021a), urban population percentage (World Population Review, 2021a), number of hospital beds, female and male lung size, median age (World Population Review, 2021b), crime index, population number (Uğur \& Akbıyık, 2020), smoking index (Ritchie \& Roser, 2013) and percentage of females (The World Bank Group, 2021). As the output factors infection rate, death rate and recovery rate were used (Kaggle, 2020).

Table 1: COVID-19 predictors

\begin{tabular}{|c|c|c|c|c|c|c|}
\hline Country & Density & $\begin{array}{c}\text { Urban } \\
\text { Population }\end{array}$ & $\begin{array}{c}\text { Hospital } \\
\text { Bed }\end{array}$ & Lung & $\begin{array}{c}\text { Female } \\
\text { Lung }\end{array}$ & $\begin{array}{l}\text { Male } \\
\text { Lung }\end{array}$ \\
\hline Albania & 105 & 63 & 2.9 & 11.67 & 7.02 & 17.04 \\
\hline Algeria & 18 & 73 & 1.9 & 8.77 & 5.03 & 12.81 \\
\hline Argentina & 17 & 93 & 5 & 29.27 & 20.16 & 42.59 \\
\hline Armenia & 104 & 63 & 4.2 & 23.86 & 16.17 & 35.99 \\
\hline Australia & 3 & 86 & 3.8 & 18.79 & 15.9 & 22.16 \\
\hline Austria & 109 & 57 & 7.6 & 17.02 & 13.02 & 22.14 \\
\hline Azerbaijan & 123 & 56 & 4.7 & 20.61 & 14.07 & 29.32 \\
\hline Bahrain & 2239 & 89 & 6.8 & 18.37 & 13.83 & 22.39 \\
\hline Bangladesh & 1265 & 39 & 0.8 & 69.07 & 61.7 & 76.24 \\
\hline Belarus & 47 & 79 & 11 & 9.46 & 2.83 & 21.51 \\
\hline Belgium & 383 & 98 & 6.2 & 27.11 & 21.2 & 34.98 \\
\hline $\begin{array}{c}\text { Bosnia and } \\
\text { Herzegovina }\end{array}$ & 64 & 52 & 3.5 & 18.48 & 12.88 & 26.05 \\
\hline Brazil & 25 & 88 & 2.2 & 26.57 & 19.71 & 35.83 \\
\hline Bulgaria & 64 & 76 & 6.8 & 19.79 & 13.98 & 27.92 \\
\hline Cambodia & 95 & 24 & 0.8 & 34.21 & 30.47 & 39.66 \\
\hline Canada & 4 & 81 & 2.7 & 19.01 & 16.95 & 21.55 \\
\hline Chile & 26 & 85 & 2.2 & 14.84 & 12.24 & 18.58 \\
\hline China & 153 & 61 & 4.2 & 63.1 & 56.35 & 70.52 \\
\hline Colombia & 46 & 80 & 1.5 & 36.3 & 31.33 & 42.49 \\
\hline
\end{tabular}




\begin{tabular}{|c|c|c|c|c|c|c|}
\hline Costa Rica & 100 & 80 & 1.2 & 20.42 & 17.65 & 23.65 \\
\hline Croatia & 73 & 58 & 5.6 & 21.75 & 14.54 & 32.7 \\
\hline Cuba & 106 & 78 & 5.2 & 23.3 & 20.53 & 26.67 \\
\hline Cyprus & 131 & 67 & 3.4 & 17.85 & 11.34 & 26.04 \\
\hline Czech Republic & 139 & 74 & 6.5 & 19.68 & 13.1 & 28.54 \\
\hline Denmark & 137 & 88 & 2.5 & 31.99 & 31.31 & 32.85 \\
\hline $\begin{array}{c}\text { Dominican } \\
\text { Republic }\end{array}$ & 225 & 85 & 1.6 & 11.43 & 9.5 & 13.57 \\
\hline Ecuador & 71 & 63 & 1.5 & 24.07 & 18.85 & 30.17 \\
\hline Estonia & 31 & 68 & 5 & 8.8 & 3.59 & 18.36 \\
\hline Ethiopia & 115 & 21 & 0.3 & 18.56 & 13.55 & 24.28 \\
\hline Finland & 18 & 86 & 4.4 & 9.92 & 5.47 & 15.97 \\
\hline France & 119 & 82 & 6.5 & 13.15 & 9.64 & 17.69 \\
\hline Georgia & 57 & 58 & 2.6 & 23.26 & 15.96 & 34.46 \\
\hline Germany & 240 & 76 & 8.3 & 20.01 & 16.32 & 24.67 \\
\hline Ghana & 137 & 57 & 0.9 & 21.09 & 17.95 & 25.11 \\
\hline Greece & 81 & 85 & 4.3 & 20.62 & 18.3 & 23.49 \\
\hline Honduras & 89 & 57 & 0.7 & 21.39 & 22.03 & 20.76 \\
\hline Hungary & 107 & 72 & 7 & 27.68 & 19.86 & 39.93 \\
\hline Iceland & 3 & 94 & 3.2 & 19.71 & 20.44 & 18.65 \\
\hline India & 464 & 35 & 0.7 & 96.92 & 87.54 & 106.89 \\
\hline Indonesia & 151 & 56 & 1.2 & 36.26 & 23.54 & 51.83 \\
\hline Ireland & 72 & 63 & 2.8 & 24.92 & 23.02 & 27.25 \\
\hline Israel & 400 & 93 & 3.1 & 16.1 & 12.81 & 20.23 \\
\hline Italy & 206 & 69 & 3.4 & 17 & 12.78 & 22.78 \\
\hline Jamaica & 273 & 55 & 1.7 & 15.74 & 6.33 & 26.44 \\
\hline Japan & 347 & 92 & 13.4 & 12.17 & 5.8 & 20.85 \\
\hline Kazakhstan & 7 & 58 & 6.7 & 114.28 & 79.27 & 174.88 \\
\hline Kenya & 94 & 28 & 1.4 & 19.15 & 14.92 & 24.16 \\
\hline South Korea & 527 & 82 & 11.5 & 10.48 & 6.6 & 16.23 \\
\hline Kuwait & 240 & 92 & 2 & 10.99 & 7.44 & 13.43 \\
\hline Latvia & 30 & 69 & 5.8 & 8.06 & 2.76 & 17.68 \\
\hline Lebanon & 667 & 78 & 2.9 & 17.28 & 13.86 & 20.78 \\
\hline Lithuania & 43 & 71 & 7.3 & 11.5 & 4.13 & 25.02 \\
\hline Luxembourg & 242 & 88 & 4.8 & 20.52 & 17.2 & 24.91 \\
\hline Malaysia & 99 & 78 & 1.9 & 23.73 & 12.95 & 34.24 \\
\hline Maldives & 1802 & 35 & 4.3 & 26.7 & 26.61 & 26.89 \\
\hline Malta & 1380 & 93 & 4.7 & 12.05 & 6.66 & 19.15 \\
\hline Mauritius & 626 & 41 & 3.4 & 30.56 & 17.36 & 48.62 \\
\hline Mexico & 66 & 84 & 1.5 & 27.85 & 23.43 & 33.14 \\
\hline Moldova & 123 & 43 & 5.8 & 17.13 & 9.21 & 29.88 \\
\hline Mongolia & 2 & 67 & 7 & 13.4 & 9.3 & 18.76 \\
\hline Montenegro & 47 & 68 & 4 & 17.6 & 10.7 & 26.47 \\
\hline
\end{tabular}




\begin{tabular}{|c|c|c|c|c|c|c|}
\hline Morocco & 83 & 64 & 1.1 & 10.39 & 7.51 & 13.73 \\
\hline Namibia & 3 & 55 & 2.7 & 43.86 & 29.39 & 65.58 \\
\hline Nepal & 203 & 21 & 0.3 & 100.75 & 94.59 & 107.64 \\
\hline Netherlands & 508 & 92 & 4.7 & 26 & 22.74 & 30.36 \\
\hline New Zealand & 18 & 87 & 2.8 & 21.88 & 19.66 & 24.61 \\
\hline Nigeria & 226 & 52 & 0.3 & 21.14 & 19.65 & 22.75 \\
\hline Norway & 15 & 83 & 3.9 & 26.13 & 23.51 & 29.49 \\
\hline Oman & 16 & 87 & 1.6 & 10.87 & 9.06 & 12.54 \\
\hline Pakistan & 287 & 35 & 0.6 & 50.61 & 35.5 & 64.92 \\
\hline Panama & 58 & 68 & 2.3 & 24.25 & 19.96 & 29.06 \\
\hline Peru & 26 & 79 & 1.6 & 17.69 & 14.94 & 20.96 \\
\hline Philippines & 368 & 47 & 1 & 44.52 & 27.04 & 67.56 \\
\hline Poland & 124 & 60 & 6.5 & 16.16 & 9.98 & 25.9 \\
\hline Portugal & 111 & 66 & 3.4 & 13.35 & 9.14 & 19.18 \\
\hline Qatar & 248 & 96 & 1.2 & 7.87 & 5.37 & 9.21 \\
\hline Romania & 84 & 55 & 6.3 & 18.8 & 10.65 & 30.05 \\
\hline Saudi Arabia & 16 & 84 & 2.7 & 12.41 & 9.55 & 15.56 \\
\hline Serbia & 100 & 56 & 5.7 & 23.27 & 15.49 & 33.82 \\
\hline Singapore & 8358 & 99 & 2.4 & 8.32 & 3.17 & 14.81 \\
\hline Slovenia & 103 & 55 & 4.6 & 11.89 & 8.36 & 16.95 \\
\hline South Africa & 49 & 67 & 2.8 & 44.3 & 28.03 & 75.32 \\
\hline Spain & 94 & 80 & 3 & 16.37 & 8.22 & 27.49 \\
\hline Sri Lanka & 341 & 18 & 3.6 & 21.58 & 15.38 & 29.36 \\
\hline Sweden & 25 & 88 & 2.6 & 16.17 & 16.58 & 17.03 \\
\hline Switzerland & 219 & 74 & 4.7 & 15.22 & 11.94 & 19.47 \\
\hline Tanzania & 67 & 37 & 0.7 & 18.09 & 14.24 & 22.47 \\
\hline Thailand & 137 & 51 & 2.1 & 25.82 & 12.91 & 41.67 \\
\hline Tunisia & 76 & 70 & 2.3 & 23.54 & 10.97 & 38.83 \\
\hline Turkey & 110 & 76 & 2.7 & 35.63 & 21.2 & 55.26 \\
\hline United States & 36 & 83 & 2.9 & 32.01 & 30.04 & 34.55 \\
\hline Ukraine & 75 & 69 & 8.8 & 11.11 & 4.73 & 22.11 \\
\hline $\begin{array}{c}\text { United Arab } \\
\text { Emirates }\end{array}$ & 118 & 86 & 1.2 & 18.37 & 12.55 & 20.21 \\
\hline United Kingdom & 281 & 83 & 2.8 & 23.66 & 21.11 & 26.84 \\
\hline Uruguay & 20 & 96 & 2.8 & 33.57 & 23.14 & 49.85 \\
\hline Vietnam & 314 & 38 & 2.6 & 26.26 & 16.8 & 41.72 \\
\hline Country & \multicolumn{2}{|c|}{$\begin{array}{c}\text { Median } \\
\text { Age }\end{array}$} & \multicolumn{2}{|c|}{\begin{tabular}{|c|} 
Population \\
2020
\end{tabular}} & $\begin{array}{c}\text { Smoking } \\
2016\end{array}$ & $\begin{array}{c}\text { Females } \\
2018 \\
\end{array}$ \\
\hline Albania & \multicolumn{2}{|c|}{32.9} & \multicolumn{2}{|c|}{2877.797} & 28.7 & 49.06309 \\
\hline Algeria & \multicolumn{2}{|c|}{28.1} & \multicolumn{2}{|c|}{43851.04} & 15.6 & 49.48427 \\
\hline Argentina & \multicolumn{2}{|c|}{31.7} & 62.96 & 45195.77 & 21.8 & 51.23735 \\
\hline Armenia & \multicolumn{2}{|c|}{35.1} & 20.78 & 2963.243 & 24.1 & 52.95658 \\
\hline Australia & 38.7 & 42.7 & \multicolumn{2}{|c|}{25499.88} & 14.7 & 50.19962 \\
\hline
\end{tabular}




\begin{tabular}{|c|c|c|c|c|c|}
\hline Austria & 44 & 23.23 & 9006.398 & 29.6 & 50.82943 \\
\hline Azerbaijan & 31.3 & 32.68 & 10139.18 & 20.8 & 50.11575 \\
\hline Bahrain & 32.3 & 29.18 & 1701.575 & 26.4 & 36.34825 \\
\hline Bangladesh & 26.7 & 64.98 & 164689.4 & 23 & 49.3873 \\
\hline Belarus & 40 & 24.8 & 9449.323 & 26.7 & 53.45605 \\
\hline Belgium & 41.4 & 42.5 & 11589.62 & 28.2 & 50.59332 \\
\hline $\begin{array}{l}\text { Bosnia and } \\
\text { Herzegovina }\end{array}$ & 42.1 & 43.57 & 3280.819 & 38.9 & 51.01054 \\
\hline Brazil & 32 & 69.48 & 212559.4 & 13.9 & 50.82992 \\
\hline Bulgaria & 42.7 & 39.31 & 6948.445 & 37 & 51.41409 \\
\hline Cambodia & 25.3 & 51.8 & 16718.97 & 17.2 & 51.19798 \\
\hline Canada & 42.2 & 39.48 & 37742.15 & 14.3 & 50.39153 \\
\hline Chile & 34.4 & 47.12 & 19116.2 & 37.8 & 50.72703 \\
\hline China & 37.4 & 36.7 & 1439324 & 25.6 & 48.67937 \\
\hline Colombia & 30 & 52.54 & 50882.89 & 9 & 50.92577 \\
\hline Costa Rica & 31.3 & 55.77 & 5094.118 & 11.9 & 50.00985 \\
\hline Croatia & 43 & 24.23 & 4105.267 & 37 & 51.85262 \\
\hline Cuba & 41.5 & 27.62 & 11326.62 & 35.2 & 50.33285 \\
\hline Cyprus & 36.8 & 29.62 & 1207.359 & 36.4 & 49.97107 \\
\hline Czech Republic & 42.1 & 25.99 & 10708.98 & 34.3 & 50.80859 \\
\hline Denmark & 42.2 & 24.72 & 5792.202 & 19.1 & 50.2742 \\
\hline $\begin{array}{c}\text { Dominican } \\
\text { Republic }\end{array}$ & 28.1 & 60.62 & 10847.91 & 13.7 & 50.0078 \\
\hline Ecuador & 27.7 & 48.91 & 17643.05 & 7.1 & 49.97063 \\
\hline Estonia & 42.7 & 22.17 & 1326.535 & 31.3 & 52.85843 \\
\hline Ethiopia & 17.9 & 47.46 & 114963.6 & 4.4 & 49.97889 \\
\hline Finland & 42.5 & 22.75 & 5540.72 & 20.4 & 50.72076 \\
\hline France & 41.4 & 46.45 & 65273.51 & 32.7 & 51.58424 \\
\hline Georgia & 38.1 & 20.18 & 3989.167 & 28.8 & 52.29124 \\
\hline Germany & 47.1 & 34.6 & 83783.94 & 30.6 & 50.66037 \\
\hline Ghana & 21.1 & 51.57 & 31072.94 & 3.9 & 49.32583 \\
\hline Greece & 44.5 & 39.29 & 10423.05 & 43.4 & 50.9162 \\
\hline Honduras & 23 & 75.84 & 9904.607 & 2 & 50.04934 \\
\hline Hungary & 42.3 & 35.41 & 9660.351 & 30.6 & 52.43243 \\
\hline Iceland & 36.5 & 23.15 & 341.243 & 14.7 & 49.81171 \\
\hline India & 27.9 & 42.38 & 1380004 & 11.5 & 48.02354 \\
\hline Indonesia & 30.2 & 46.26 & 273523.6 & 39.4 & 49.64388 \\
\hline Ireland & 36.8 & 46.18 & 4937.786 & 24.3 & 50.42551 \\
\hline Israel & 29.9 & 30.71 & 8655.535 & 25.2 & 50.29813 \\
\hline Italy & 45.5 & 44.35 & 60461.83 & 23.7 & 51.37667 \\
\hline Jamaica & 26 & 65.26 & 2961.167 & 16.8 & 50.33983 \\
\hline Japan & 47.3 & 15.91 & 126476.5 & 22.1 & 51.15926 \\
\hline Kazakhstan & 30.6 & 64.23 & 18776.71 & 24 & 51.51148 \\
\hline
\end{tabular}


TOURISM CHALLENGES AMID COVID-19 (TISC 2021) - Thematic proceedings

\begin{tabular}{|c|c|c|c|c|c|}
\hline Kenya & 19.7 & 62.38 & 53771.3 & 10.7 & 50.31602 \\
\hline South Korea & 41.8 & 29.24 & 51269.19 & 23.3 & 49.91688 \\
\hline Kuwait & 29.3 & 35.61 & 4270.571 & 22.5 & 39.54817 \\
\hline Latvia & 43.6 & 36.6 & 1886.198 & 37 & 54.01017 \\
\hline Lebanon & 30.5 & 43.38 & 6825.445 & 33.8 & 49.70581 \\
\hline Lithuania & 43.7 & 34.82 & 2722.289 & 28.8 & 53.79196 \\
\hline Luxembourg & 39.3 & 30.17 & 625.978 & 23.5 & 49.53926 \\
\hline Malaysia & 28.5 & 60.66 & 32366 & 21.5 & 48.57852 \\
\hline Maldives & 28.2 & 53.83 & 540.544 & 28.3 & 37.26498 \\
\hline Malta & 41.8 & 37.73 & 441.543 & 25.5 & 49.87331 \\
\hline Mauritius & 35.3 & 47.34 & 1271.768 & 21.6 & 50.57711 \\
\hline Mexico & 28.3 & 52.51 & 128932.8 & 14 & 51.08928 \\
\hline Moldova & 36.7 & 45.7 & 4033.963 & 24.2 & 52.03556 \\
\hline Mongolia & 28.3 & 57.76 & 3278.29 & 25.6 & 50.66954 \\
\hline Montenegro & 40.7 & 39.67 & 628.066 & 45.9 & 50.55901 \\
\hline Morocco & 29.3 & 49.53 & 36910.56 & 23.4 & 50.40385 \\
\hline Namibia & 21.2 & 68.14 & 2540.905 & 21.4 & 51.55369 \\
\hline Nepal & 24.1 & 35.7 & 29136.81 & 22.8 & 54.53534 \\
\hline Netherlands & 42.6 & 28.54 & 17134.87 & 25.8 & 50.22094 \\
\hline New Zealand & 37.9 & 40.89 & 4822.233 & 16 & 50.83777 \\
\hline Nigeria & 18.4 & 64.64 & 206139.6 & 5.8 & 49.33611 \\
\hline Norway & 39.2 & 33.51 & 5421.241 & 20.2 & 49.52463 \\
\hline Oman & 25.6 & 21.55 & 5106.626 & 11.1 & 34.01408 \\
\hline Pakistan & 23.8 & 44.58 & 220892.3 & 20.1 & 48.53807 \\
\hline Panama & 29.2 & 45.47 & 4314.767 & 6.1 & 49.90538 \\
\hline Peru & 28 & 64.58 & 32971.85 & 4.8 & 50.33776 \\
\hline Philippines & 23.5 & 41.09 & 109581.1 & 24.3 & 49.74166 \\
\hline Poland & 40.7 & 29.67 & 37846.61 & 28 & 51.53071 \\
\hline Portugal & 42.2 & 30.11 & 10196.71 & 22.7 & 52.71196 \\
\hline Qatar & 33.2 & 12 & 2881.053 & 20.6 & 24.49529 \\
\hline Romania & 41.1 & 27.84 & 19237.69 & 29.7 & 51.34374 \\
\hline Saudi Arabia & 27.5 & 28.22 & 34813.87 & 15.6 & 42.44585 \\
\hline Serbia & 42.6 & 37.63 & 8737.371 & 38.9 & 51.00252 \\
\hline Singapore & 34.6 & 27.7 & 5850.342 & 16.5 & 47.65813 \\
\hline Slovenia & 44.5 & 22.01 & 2078.938 & 22.5 & 50.24521 \\
\hline South Africa & 27.1 & 77.02 & 59308.69 & 20.3 & 50.69415 \\
\hline Spain & 42.7 & 31.07 & 46754.78 & 29.3 & 50.89664 \\
\hline Sri Lanka & 32.8 & 40.15 & 21413.25 & 13 & 51.96682 \\
\hline Sweden & 41.2 & 47.21 & 10099.27 & 18.8 & 49.94578 \\
\hline Switzerland & 42.4 & 21.18 & 8654.622 & 25.7 & 50.42712 \\
\hline Tanzania & 17.7 & 59.83 & 59734.22 & 14.8 & 50.05101 \\
\hline Thailand & 37.7 & 41.29 & 69799.98 & 19.9 & 51.2687 \\
\hline Tunisia & 31.6 & 40.64 & 11818.62 & 32.7 & 50.43715 \\
\hline
\end{tabular}




\begin{tabular}{|c|c|c|c|c|c|}
\hline Turkey & 30.9 & 39.86 & 84339.07 & 27.2 & 50.67781 \\
\hline United States & 38.1 & 46.73 & 331002.7 & 21.8 & 50.52001 \\
\hline Ukraine & 40.6 & 49.04 & 43733.76 & 28.9 & 53.68775 \\
\hline $\begin{array}{c}\text { United Arab } \\
\text { Emirates }\end{array}$ & 30.3 & 15.52 & 9890.402 & 28.9 & 30.63669 \\
\hline United Kingdom & 40.5 & 43.64 & 67886.01 & 22.3 & 50.63527 \\
\hline Uruguay & 35 & 52.33 & 3473.73 & 16.8 & 51.72154 \\
\hline Vietnam & 30.5 & 48.22 & 97338.58 & 22.8 & 50.09641 \\
\hline
\end{tabular}

Source: Kaggle (2020)

Table 2: Output parameters for COVID-19

\begin{tabular}{|c|c|c|c|c|c|c|c|}
\hline Country & $\begin{array}{c}\text { Total } \\
\text { Infected }\end{array}$ & $\begin{array}{c}\text { Total } \\
\text { Deaths }\end{array}$ & $\begin{array}{c}\text { Total } \\
\text { Recovered }\end{array}$ & Country & $\begin{array}{c}\text { Total } \\
\text { Infected }\end{array}$ & $\begin{array}{c}\text { Total } \\
\text { Deaths }\end{array}$ & $\begin{array}{c}\text { Total } \\
\text { Recovered }\end{array}$ \\
\hline Albania & 949 & 31 & 742 & Kuwait & 16764 & 121 & 4681 \\
\hline Algeria & 7377 & 561 & 3746 & Latvia & 1012 & 21 & 694 \\
\hline Argentina & 8809 & 393 & 2872 & Lebanon & 954 & 26 & 251 \\
\hline Armenia & 5041 & 64 & 2164 & Lithuania & 1562 & 60 & 1025 \\
\hline Australia & 7072 & 100 & 6431 & Luxembourg & 3958 & 109 & 3718 \\
\hline Austria & 16321 & 632 & 14678 & Malaysia & 6978 & 114 & 5646 \\
\hline Azerbaijan & 3518 & 41 & 2198 & Maldives & 1143 & 4 & 91 \\
\hline Bahrain & 7532 & 12 & 2952 & Malta & 569 & 6 & 460 \\
\hline Bangladesh & 25121 & 370 & 4993 & Mauritius & 332 & 10 & 322 \\
\hline Belarus & 31508 & 175 & 10620 & Mexico & 54346 & 5666 & 37325 \\
\hline Belgium & 55791 & 9108 & 14687 & Moldova & 6340 & 221 & 2508 \\
\hline $\begin{array}{c}\text { Bosnia and } \\
\text { Herzegovina }\end{array}$ & 2321 & 134 & 1522 & Mongolia & 140 & 0 & 26 \\
\hline Brazil & 271885 & 17983 & 106794 & Montenegro & 324 & 9 & 312 \\
\hline Bulgaria & 2259 & 112 & 646 & Morocco & 7023 & 193 & 3901 \\
\hline Cambodia & 122 & 0 & 122 & Namibia & 16 & 0 & 13 \\
\hline Canada & 80493 & 6028 & 40069 & Nepal & 402 & 2 & 37 \\
\hline Chile & 49579 & 509 & 21507 & Netherlands & 44449 & 5734 & 167 \\
\hline China & 84063 & 4638 & 79310 & New Zealand & 1503 & 21 & 1447 \\
\hline Colombia & 16935 & 613 & 4050 & Nigeria & 6401 & 192 & 1734 \\
\hline Costa Rica & 882 & 10 & 577 & Norway & 8267 & 233 & 32 \\
\hline Croatia & 2232 & 96 & 1967 & Oman & 5671 & 27 & 1574 \\
\hline Cuba & 1887 & 79 & 1538 & Pakistan & 43966 & 939 & 12489 \\
\hline Cyprus & 918 & 17 & 515 & Panama & 9867 & 281 & 6194 \\
\hline $\begin{array}{c}\text { Czech } \\
\text { Republic }\end{array}$ & 8647 & 302 & 5726 & Peru & 99483 & 2914 & 36524 \\
\hline Denmark & 11242 & 551 & 9614 & Philippines & 12942 & 837 & 2843 \\
\hline $\begin{array}{c}\text { Dominican } \\
\text { Republic }\end{array}$ & 13223 & 441 & 6613 & Poland & 19268 & 948 & 7903 \\
\hline Ecuador & 34151 & 2839 & 3457 & Portugal & 29432 & 1247 & 6431 \\
\hline
\end{tabular}




\begin{tabular}{|c|c|c|c|c|c|c|c|}
\hline Estonia & 1791 & 64 & 938 & Qatar & 35606 & 15 & 5634 \\
\hline Ethiopia & 365 & 5 & 120 & Romania & 17191 & 1137 & 10166 \\
\hline Finland & 6399 & 301 & 5000 & Saudi Arabia & 59854 & 329 & 31634 \\
\hline France & 180933 & 28025 & 62678 & Serbia & 10733 & 234 & 4904 \\
\hline Georgia & 707 & 12 & 456 & Singapore & 28794 & 22 & 10365 \\
\hline Germany & 177778 & 8081 & 155681 & Slovenia & 1467 & 104 & 1338 \\
\hline Ghana & 6096 & 31 & 1773 & South Africa & 17200 & 312 & 7960 \\
\hline Greece & 2840 & 165 & 1374 & Spain & 232037 & 27778 & 0 \\
\hline Honduras & 2955 & 147 & 349 & Sri Lanka & 1027 & 9 & 569 \\
\hline Hungary & 3556 & 467 & 1412 & Sweden & 30799 & 3743 & 4971 \\
\hline Iceland & 1802 & 10 & 1789 & Switzerland & 30618 & 1891 & 27700 \\
\hline India & 106475 & 3302 & 42309 & Tanzania & 509 & 21 & 183 \\
\hline Indonesia & 18496 & 1221 & 4467 & Thailand & 3033 & 56 & 2857 \\
\hline Ireland & 24251 & 1561 & 19470 & Tunisia & 1044 & 47 & 826 \\
\hline Israel & 16659 & 278 & 13435 & Turkey & 151615 & 4199 & 112895 \\
\hline Italy & 226699 & 32169 & 129401 & United States 1528568 & 91921 & 289392 \\
\hline Jamaica & 520 & 9 & 145 & Ukraine & 18876 & 548 & 5632 \\
\hline Japan & 16367 & 768 & 11564 & $\begin{array}{c}\text { United Arab } \\
\text { Emirates }\end{array}$ & 25063 & 227 & 10791 \\
\hline Kazakhstan & 6751 & 35 & 3598 & $\begin{array}{c}\text { United } \\
\text { Kingdom }\end{array}$ & 250138 & 35422 & 1099 \\
\hline Kenya & 963 & 50 & 358 & Uruguay & 738 & 20 & 579 \\
\hline South Korea & 11110 & 263 & 10066 & Vietnam & 324 & 0 & 263 \\
\hline
\end{tabular}

Source: Kaggle (2020)

\section{ANFIS methodology}

ANFIS network is shown in Figure 1 where five layers can be observed. Fuzzy inference system is the main core of the network. The main principle of the network is to include input and output data samples and to train the network.

Figure 1: ANFIS

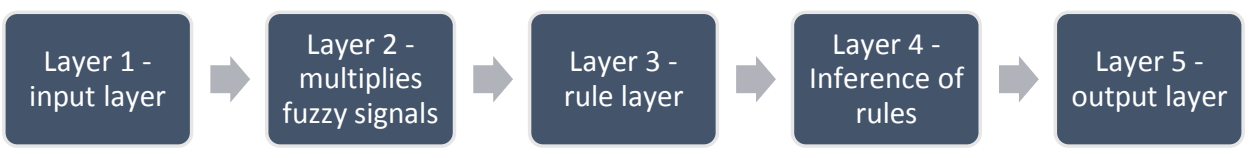

Source: Jang (1993) 


\section{Results}

The most influential variables for the TF-IDF were chosen using the ANFIS methodology. The selection is crucial, as is the preprocessing of the input parameters to exclude irrelevant inputs. The data collection is organized based on Table 1's data file. Following the commands in MATLAB Software, the dataset is partitioned into a training set (oddindexed samples) and a checking set (even-indexed samples):

[data $]=$ TF-IDF;

trn_data $=$ data $(1: 2:$ end, $:)$;

chk_data $=\operatorname{data}(2: 2:$ end, $:)$;

The function "exhsrch" conducts an exhaustive search of the available inputs to identify the collection of inputs that have the greatest effect on the TF-IDF. The function's first parameter determines the number of input combinations that will be tested during the selection process. In essence, "exhsrch" creates an ANFIS model for each combination, trains it for one epoch, and then reports the results. The command line below is used to evaluate the single most important attribute in predicting the outcome:

exhsrch(1,trn_data,chk_data);

The following results are obtained

ANFIS model 1: in1 $-->\operatorname{trn}=4.7729$, chk $=4.0893$

ANFIS model 2: in2 - $>$ trn $=20.3109$, chk $=16.3539$

ANFIS model 3: in3 - $>$ trn $=20.2467$, chk $=15.9928$

In terms of the output, the input variable 1 has the least error, or in other words, the most significance. The results show that the input attribute "Frequency" has the greatest impact on the TF-IDF. There is no overfitting since the preparation and checking errors are equivalent. The ANFIS decision surfaces for TF-IDF based on the three factors are shown in Figures 2-4. 
Figure 2: ANFIS prediction of TF-IDF based on frequency and number of cases

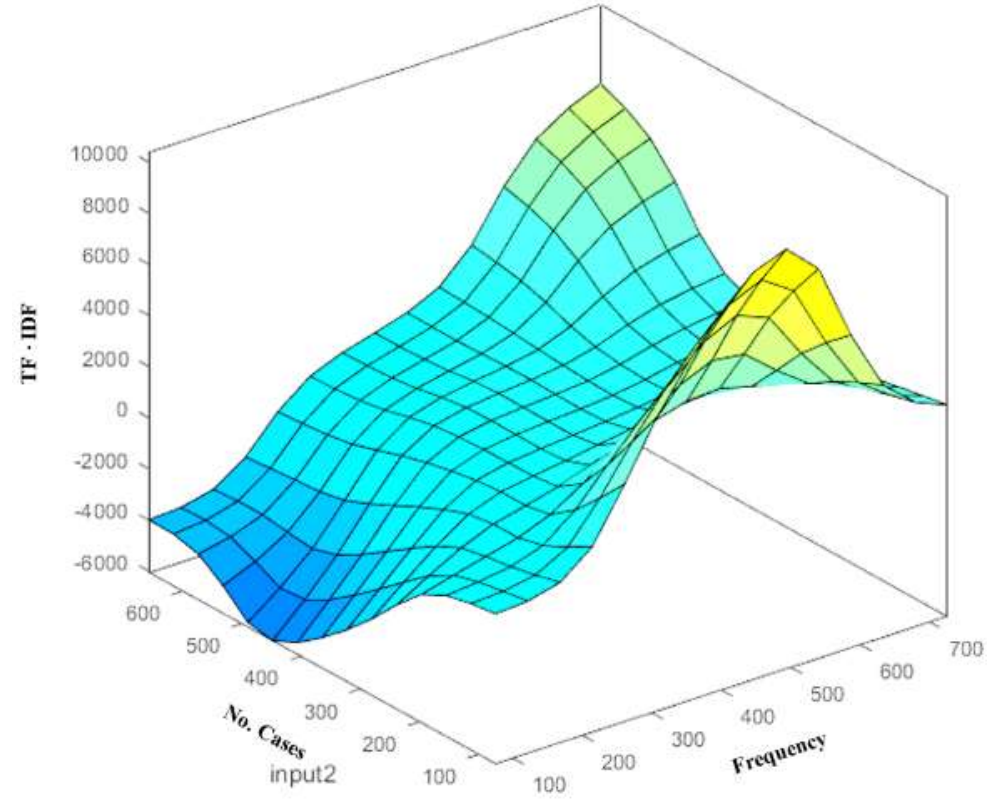

Source: Authors'own work

Figure 3: ANFIS prediction of TF-IDF based on frequency and cases percentage

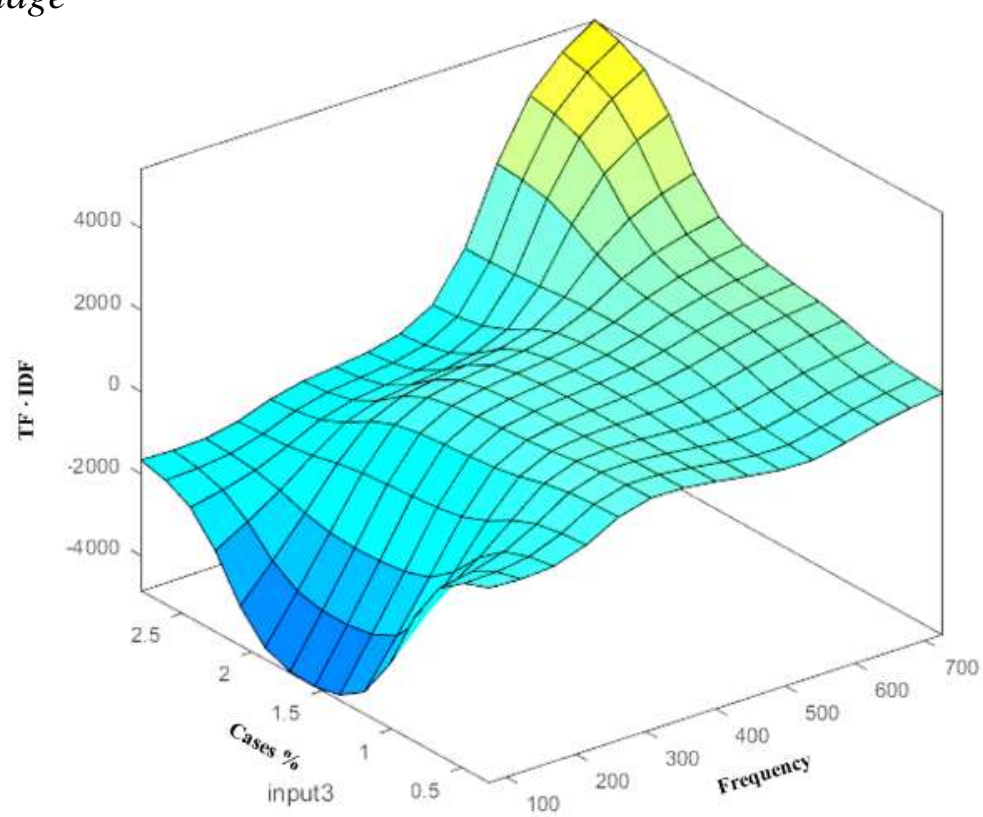

Source: Authors' own work 
Figure 4: ANFIS prediction of TF-IDF based on number of cases and cases percentage

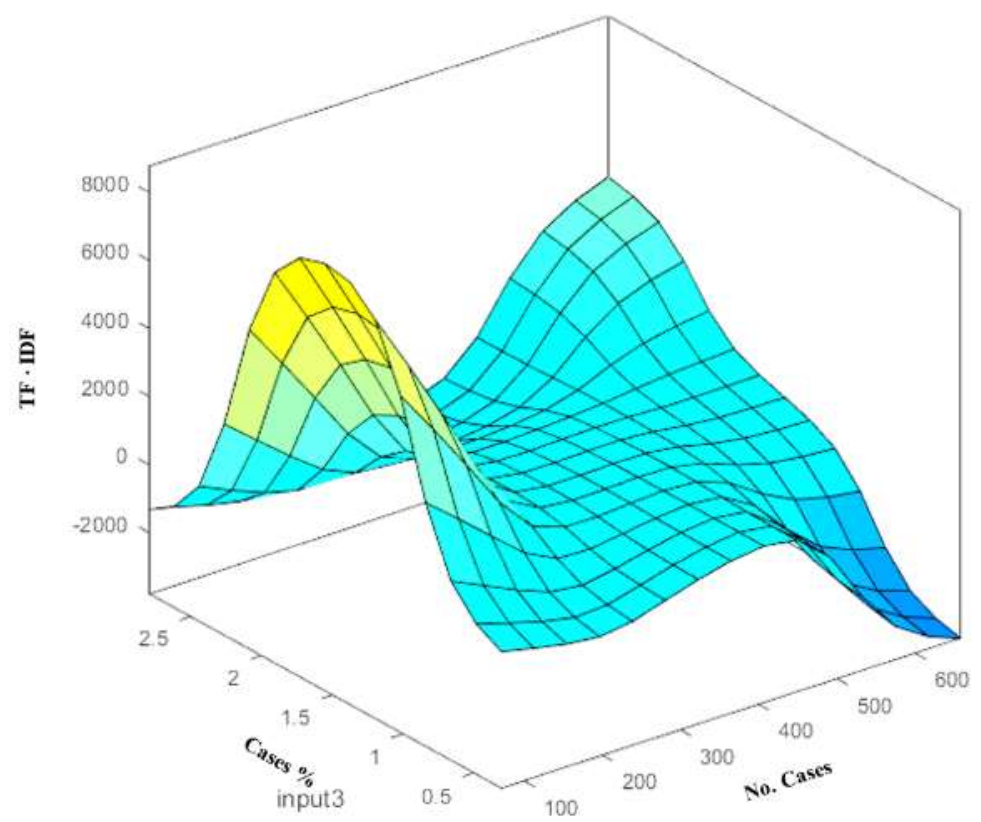

Source: Authors' own work

\section{COVID-19 predictors selection}

According to training error (trn) in Table 3 population number in 2020 (bold value) has the strongest impact on infection rate. Checking (chk) error is used for tracking of overfitting between training and checking data. The same observations can be noted in Tables 4 and 5 for death rate and recovery rate prediction. Therefore number of population is the strongest single predictor for COVID-19.

Table 3: COVID-19 predictors' accuracy for infection rate

\begin{tabular}{|l|}
\hline ANFIS model 1: Density --> trn=221712.2483, chk $=173428.0136$ \\
\hline ANFIS model 2: Urban Population --> trn=217111.7985, chk=79355.5141 \\
\hline ANFIS model 3: Hospital Bed --> trn=222877.0509, chk=59218.0037 \\
\hline ANFIS model 4: Lung --> trn=219926.4973, chk=71736.6272 \\
\hline ANFIS model 5: Female Lung --> trn=217976.8181, chk=63537.7839 \\
\hline ANFIS model 6: Male Lung --> trn=220320.5213, chk=118569.4758 \\
\hline ANFIS model 7: Median Age --> trn=218230.4643, chk=78625.0855 \\
\hline ANFIS model 8: Crime Index --> trn=220665.8308, chk=60387.4698 \\
\hline
\end{tabular}


ANFIS model 9: Population 2020 --> trn=82662.2518, chk=436987.4859

ANFIS model 10: Smoking 2016 --> trn=220159.9990, chk=72984.8979

ANFIS model 11: Females 2018 --> trn=222482.2861, chk=65735.7301

Source: Authors' own work

Table 4: COVID-19 predictors' accuracy for death rate

\begin{tabular}{|c|}
\hline ANFIS model 2: Urban Population --> trn $=14098.2589$, chk $=7508.5184$ \\
\hline ANFIS model 3: Hospital Bed --> trn=14625.7927, chk=6237.5719 \\
\hline ANFIS model 4: Lung --> trn=14595.9762, chk=6070.9450 \\
\hline ANFIS model 5: Female Lung --> trn=14539.0685, chk=5715.5738 \\
\hline ANFIS model 6: Male Lung --> trn=14546.6911, chk=7819.8976 \\
\hline ANFIS model 7: Median Age $-->\operatorname{trn}=14194.7566$, chk=7082.8291 \\
\hline ANFIS model 8: Crime Index --> trn=14476.0085, chk=5897.0950 \\
\hline ANFIS model 9: Population 2020 --> trn=8314.3409, chk=25039.1436 \\
\hline ANFIS model 10: Smoking $2016-->$ trn $=14442.2460, \mathrm{chk}=6845.0943$ \\
\hline ANFIS model 11: Females 2018 --> trn=14573.2227, chk=7057.1682 \\
\hline
\end{tabular}

Source: Authors' own work

Table 5: COVID-19 predictors' accuracy for recovery rate

ANFIS model 1: Density --> trn=50722.7634, chk=24905.3444

ANFIS model 2: Urban Population --> trn=48831.7813, chk $=26000.0839$

ANFIS model 3: Hospital Bed --> trn=50058.7364, chk=29588.6144

ANFIS model 4: Lung --> trn=50372.4841, chk=27090.3174

ANFIS model 5: Female Lung --> trn=50056.1160, chk=23182.5764

ANFIS model 6: Male Lung --> trn=50307.3893, chk=38889.7417

ANFIS model 7: Median Age --> trn=49433.9358, chk=25140.0393

ANFIS model 8: Crime Index --> trn=50382.1178, chk=22497.1108

ANFIS model 9: Population 2020 --> trn=31874.6665, chk=74489.6323

ANFIS model 10: Smoking 2016 --> trn=50280.8124, chk=23877.6985

ANFIS model 11: Females 2018 --> trn=50654.2781, chk=24425.0319

Source: Authors' own work

\section{Conclusion}

The infection of Coronavirus Disease 2019 (COVID-19) has now spread worldwide, affecting over a million people. To reduce morbidity and social 
burden, predictors of disease outcomes in these patients must be evaluated as soon as possible.

In this study, the effect of eleven predictors for COVID-19 was analyzed. The main concluding remarks are:

- The combination of number of hospital beds, population number and smoking index is the optimal combination for the best prediction of infection rate of COVID-19.

- The optimal combination of the predictors for the death rate is number of hospital beds, median age and population number.

- The combination of median age, crime index and population number is the most impactful combination for the death rate of COVID-19.

The results revealing the frequency of the used words in the pandemic show the highest impact on the travelers' reactions. The travel industry was already having problems. Many businesses have mastered the complexities of digitization and thrived in a rapidly evolving world. The scale of Covid19 's effects, on the other hand, is not comparable to previous crises. The demand for mobility, consumption, and independence is expected to remain strong in the future, but it will be shaped by local considerations.

\section{Acknowledgements}

The realization of the research was financed from the budget of the Republic of Serbia, based on the decision of the Ministry of Education, Science and Technological Development on financing scientific research work in 2021, number: 451-03-9 / 2021-14.

\section{References}

1. Fabio, C., Antonella, C., Patrizia, R. Q., Annalisa, R., Laura, G., Caterina, C.,... Maria, F. (2020). Early predictors of clinical outcomes of COVID-19 outbreak in Milan, Italy. Clinical Immunology, Vol. 217, 108509.

2. Hasan, N. (2020). A Methodological Approach for Predicting COVID19 Epidemic Using EEMD-ANN Hybrid Model. Internet of Things, Vol. $11,100228$.

3. Henry, B. M., Aggarwal, G., Wong, J., Benoit, S., Vikse, J., Plebani, M., Lippi, G. (2020). Lactate dehydrogenase levels predict coronavirus disease 2019 (COVID-19) severity and mortality: A pooled analysis. The American Journal of Emergency Medicine, Vol. 38, No. 9, 1722-1726. 
4. Jang, J.S.R, (1993). ANFIS: Adaptive-Network-based Fuzzy Inference Systems. IEEE Trans. On Systems, Man, and Cybernetics, Vol. 23, No. 3, 665-685.

5. Kaggle, (2020), Country info, https://www.kaggle.com/koryto/ countryinfo, (10 February 2021).

6. Laković, N., Khan, A., Petković, B., Petkovic, D., Kuzman, B., Resic, S.,... Azam, S. (2021). Management of higher heating value sensitivity of biomass by hybrid learning technique. Biomass Conversion and Biorefinery, 1-8.

7. Milić, M., Petković, B., Selmi, A., Petković, D., Jermsittiparsert, K., Radivojević, A., ... Kuzman, B. (2021). Computational evaluation of microalgae biomass conversion to biodiesel. Biomass Conversion and Biorefinery, 1-8.

8. Petković, B., Petković, D., Kuzman, B. (2020a). Adaptive neuro fuzzy predictive models of agricultural biomass standard entropy and chemical exergy based on principal component analysis. Biomass Conversion and Biorefinery, 1-11.

9. Petković, B., Petković, D., Kuzman, B., Milovančević, M., Wakil, K., Ho, L. S., Jermsittiparsert, K. (2020b). Neuro-fuzzy estimation of reference crop evapotranspiration by neuro fuzzy logic based on weather conditions. Computers and Electronics in Agriculture, Vol. 173, 105358.

10. Petković, D., Petković, B., Kuzman, B. (2020c). Appraisal of information system for evaluation of kinetic parameters of biomass oxidation. Biomass Conversion and Biorefinery, 1-9.

11. Ritchie, H., Roser, M. (2013). Smoking, https://ourworldindata.org/ smoking\#prevalence-of-smoking-across-the-world, (15 February 2021).

12. Sun, D. W., Zhang, D., Tian, R. H., Li, Y., Wang, Y. S., Cao, J.,... Huang, Y. Z. (2020a). The underlying changes and predicting role of peripheral blood inflammatory cells in severe COVID-19 patients: a sentinel?. Clinica Chimica Acta, Vol. 508, 122-129.

13. Sun, L., Liu, G., Song, F., Shi, N., Liu, F., Li, S.,... Sun, L. (2020b). Combination of four clinical indicators predicts the severe/critical 
symptom of patients infected COVID-19. Journal of Clinical Virology, Col. 128, 104431.

14. Tamara, A., Tahapary, D. L. (2020). Obesity as a predictor for a poor prognosis of COVID-19: A systematic review. Diabetes \& Metabolic Syndrome: Clinical Research \& Reviews, Vol. 14, No. 4. 655-659.

15. The World Bank Group, (2021), Population, female (\% of total population), https://data.worldbank.org/indicator/SP.POP.TOTL.FE.ZS, (10 January 2021).

16. Uğur, N. G., Akbiyık, A. (2020). Impacts of COVID-19 on global tourism industry: A cross-regional comparison. Tourism Management Perspectives, Vol. 36, 100744.

17. Wang, F., Hou, H., Wang, T., Luo, Y., Tang, G., Wu, S.,... Sun, Z. (2020a). Establishing a model for predicting the outcome of COVID-19 based on combination of laboratory tests. Travel Medicine and Infectious Disease, Vol. 36, 101782.

18. Wang, H., Zhang, Y., Mo, P., Liu, J., Wang, H., Wang, F., Zhao, Q. (2020b). Neutrophil to CD4+ lymphocyte ratio as a potential biomarker in predicting virus negative conversion time in COVID-19. International Immunopharmacology, Vol. 85, 106683.

19. World Population Review, (2021a), Crime Rate by Country 2021, https://worldpopulationreview.com/countries/crime-rate-by-country/, (11 January 2021).

20. World Population Review, (2021b), Median Age 2021, https://world populationreview.com/countries/median-age/, (21 January 2021).

21. Xie, J., Shi, D., Bao, M., Hu, X., Wu, W., Sheng, J.,... Fang, D. (2020). A predictive nomogram for predicting improved clinical outcome probability in patients with COVID-19 in zhejiang province, china. Engineering, (article in press).

22. Zhang, X., Ma, R., Wang, L. (2020). Predicting turning point, duration and attack rate of COVID-19 outbreaks in major Western countries. Chaos, Solitons \& Fractals, Vol. 135, 109829. 